\title{
New lifelike mouth models to help dental students
}

Dental students' training will be enhanced thanks to the use of new dental models with tactile qualities of real mouths, it has been announced.

The models, featuring realistic gums as well as tongues that feel and behave like the real thing, are designed to allow students to learn how to examine the mouth safely and check for disease.

The project is the result of research by Nottingham Trent University (NTU) and the University of Birmingham.

A joint project between NTU researcher Richard Arm and colleagues Michael Milward and Paul Cooper from the University of Birmingham's School of Dentistry, it will allow student dentists to experience and learn how it feels to use periodontal probes to check for gum disease.

Funded by the University of Birmingham's Alumni Impact Fund, the models are made from synthetic gels and fibres and the tongue and gums vary in hardness to mimic living tissue while the teeth and jaw bones are made from bone-simulating resin.

$\mathrm{PhD}$ candidate Mr Arm of the Advanced Textiles Research Group at NTU's School of Art \& Design, said: 'The aim is to give students the psychological experience of how it feels to perform real dentistry, but in a safe learning environment. These realistic models will allow students to learn the tactile skills of how to examine the gums and teeth, before examining their first patients.

'Until now current dental models haven't provided a realistic enough experience for students and the inclusion of a tongue will

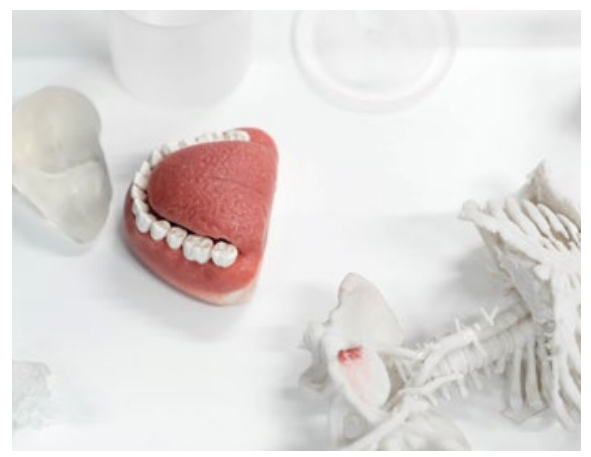

mimic the challenge which dentists face and better prepare them for their first clinic.'

Students and staff from the University of Birmingham's School of Dentistry have provided feedback on prototype dental training models to help inform the final design.

Dr Michael Milward, Reader and

Honorary Consultant in Clinical Periodontology at the University of Birmingham, said: 'These models meet an unmet need in dental education and will allow us to better prepare our students for clinical work.

'The feedback we have received from students and staff has been extremely positive and the final version has already been introduced into undergraduate teaching. While some models are commercially available, no models combine the replica hard and soft tissues in this way to provide a realistic learning experience.

'These developments provide a major step forward in dental education both for dental students and for the retraining of the dental workforce, ultimately benefitting our patients.' 